\title{
Hypertriglyceridemia with acute pancreatitis in a 14-year-old girl with diabetic ketoacidosis
}

\author{
Hyojung Park', \\ Min-Sun Kim², \\ Jiyeon $\mathrm{Kim}^{2}$, \\ Sae-Mi Lee ${ }^{3,4}$, \\ Sung Yoon $\mathrm{Cho}^{2}$, \\ Eun-Gyong Yoo ${ }^{5}$, \\ Dong-Kyu Jin ${ }^{2}$
}

'Department of Pediatrics, Seongnam Citizens Medical Center, Seongnam, Korea

${ }^{2}$ Department of Pediatrics, Samsung Medical Center, Sungkyunkwan University School of Medicine, Seoul, Korea ${ }^{3}$ GC Genome, GCLabs, Yongin, Korea ${ }^{4}$ Department of Laboratory Medicine, Kangwon National University School of Medicine, Chuncheon, Korea

${ }^{5}$ Department of Pediatrics, College of Medicine, Pochon CHA University, Seongnam, Korea

\begin{abstract}
Diabetic ketoacidosis (DKA) is a medically fatal condition in poorly controlled hyperglycemia or newly diagnosed diabetes mellitus. Severe hypertriglyceridemia (HTG) is an uncommon complication of DKA and can be associated with acute pancreatitis (AP). We present the clinical manifestations, laboratory findings, and management of AP associated with HTG in a 14-year-old girl with DKA. The patient, with a 7-year history of type 2 diabetes presented with epigastric pain, 1 month after stopping insulin injection. DKA, severe HTG, and AP were diagnosed based on the laboratory and imaging tests. She recovered from DKA after conventional treatment for DKA, and her triglyceride (TG) level was reduced from 10,867 mg/ $\mathrm{dL}$ to the normal range after 7 days of admission without antilipid medication. Given that her C-peptide level was not too low and considering her negative diabetes-related antibodies and high TG level, targeted gene panel sequencing was performed on the genes associated with diabetes and HTG. We identified a heterozygous mutation, c.4607C $>T$ (p. Ala1537Val), in $A B C C 8$ related to maturityonset diabetes of the young (MODY) 12. To our knowledge, this is the first reported case of HTG-induced AP with DKA in a patient with MODY. In addition, we reviewed the literature for pediatric cases of HTG with DKA. In patients with DKA, timely awareness of severe HTG related to insulin deficiency is crucial for improving the consequences of AP. We recommend considering AP in all DKA patients presenting with severe HTG to ensure early and proper management.
\end{abstract}

Keywords: Hypertriglyceridemia, Acute pancreatitis, Diabetic ketoacidosis

\section{Highlights}

- Severe HTG is an uncommon complication of DKA and can be associated with AP. In patients with DKA, timely awareness of severe HTG related to insulin deficiency is crucial for improving the consequences of AP.

\section{Introduction}

Received: 16 November, 2020

Revised: 5 January, 2021

Accepted: 1 February, 2021

Address for correspondence:

Sung Yoon Cho

Department of Pediatrics, Samsung Medical Center, Sungkyunkwan University School of Medicine, 81 Irwon-ro, Gangnam-gu, Seoul 06351, Korea

Email:nadri1217@naver.com

https://orcid.org/0000-0003-2913059X
Diabetic ketoacidosis (DKA) is a medically fatal condition that may occur in patients with poorly controlled hyperglycemia or newly diagnosed diabetes mellitus (DM). Cerebral edema is its most devastating complication and causes $>20 \%$ of deaths among DKA cases. ${ }^{1)}$ Severe hypertriglyceridemia (HTG, triglyceride $[\mathrm{TG}]>1,000 \mathrm{mg} / \mathrm{dL}$ ) is an uncommon complication of DKA and can be associated with acute pancreatitis (AP). ${ }^{2)}$ The combination of DKA, HTG, and AP has been discussed in adults. While severe HTG was identified in around $8 \%$ of adult DKA cases, data of this combination in children have remained limited. ${ }^{3,4)}$ Here, we present the clinical manifestations, laboratory findings, and management of AP associated with HTG in a 14-year-old girl with DKA. In addition, literature on HTG with DKA in pediatric cases was reviewed. 


\section{Case report}

A 14-year-old girl presented to Samsung Medical Center owing to severe epigastric pain, nausea, and fever for 1 day. She was diagnosed with type $2 \mathrm{DM}$ at another hospital at the age of 7 years and 10 months, when her hemoglobin $\mathrm{Alc}(\mathrm{HbAlc})$ was $10.1 \%$ and postprandial C-peptide level was $7.2 \mathrm{ng} / \mathrm{mL}$, while her diabetes-related antibodies were all negative. She was treated with metformin ( $\leq 2 \mathrm{~g} /$ day) in the early stage of DM; however, basal insulin (glargine) was added to metformin owing to the poor control of hyperglycemia (HbAlc level, 14.0\%). It was 1 month before this episode that she discontinued blood glucose testing and regular insulin injections. When she presented to our hospital, her mental status was alert and vital signs included a blood pressure of 132/70 mmHg, heart rate of $157 \mathrm{bpm}$, respiratory rate of 24 breaths/min, and body temperature of $38.2^{\circ} \mathrm{C}$. Her body weight, height, and body mass index were 60.1 $\mathrm{kg}$ (standard deviation score [SDS], 1.18), $157.4 \mathrm{~cm}$ (SDS, -0.07), and $24.3 \mathrm{~kg} / \mathrm{m}^{2}$ (SDS, 1.38), respectively. She showed a dry mouth and decreased skin turgor. Her abdomen was soft and distended, and the bowel sounds were normal. She complained of tender epigastrium on palpation. Her laboratory findings were suggestive of DKA, such as a glucose level of $311 \mathrm{mg} / \mathrm{dL}$, venous blood gas with a pH of 7.2, $\mathrm{pCO}_{2}$ of $21 \mathrm{mmHg}, \mathrm{HCO}_{3}{ }^{-}$of 8.2 $\mathrm{mmol} / \mathrm{L}$, base excess of $-17.8 \mathrm{mmol} / \mathrm{L}$, and $\beta$-hydroxybutyrate level of $3.4 \mathrm{mmol} / \mathrm{L}$ (normal reference $<0.4-0.5 \mathrm{mmol} / \mathrm{L}$ ). Her HbAlc level was $14.2 \%$, and urinalysis revealed $3+$ glucose and $3+$ ketones. Other laboratory findings included the following results: white blood cell count of $12,380 / \mu \mathrm{L}$, hemoglobin level of $13.5 \mathrm{~g} / \mathrm{dL}$, platelet count of $205,000 / \mu \mathrm{L}$, total cholesterol level of $336 \mathrm{mg} / \mathrm{dL}$, high-density lipoprotein (HDL) level of $14 \mathrm{mg} /$

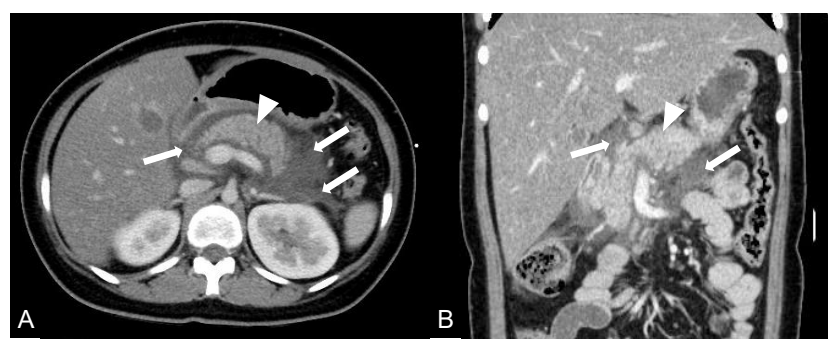

Fig. 1. Contrast-enhanced abdominal computed tomography (CT) imaging. The initial CT scan shows edematous pancreas (arrowhead) and adjacent fluid collection (arrows), suggesting grade D acute pancreatitis. (A) Axial image, (B) coronal image.
$\mathrm{dL}$, TG level of 10,867 mg/dL, low-density lipoprotein (LDL) level of $32 \mathrm{mg} / \mathrm{dL}$, aspartate aminotransferase level of $16 \mathrm{U} / \mathrm{L}$, alanine aminotransferase level of $19 \mathrm{U} / \mathrm{L}$, amylase level of 711.4 $\mathrm{U} / \mathrm{L}$ (normal reference, 28-100 U/L), lipase level of 2,403.2 U/ $\mathrm{L}$ (normal reference, 13-60 U/L), sodium level of $133 \mathrm{mmol} /$ $\mathrm{L}$, potassium level of $3.9 \mathrm{mmol} / \mathrm{L}$, chloride level of $97 \mathrm{mmol} / \mathrm{L}$, and C-reactive protein (CRP) level of $1.61 \mathrm{mg} / \mathrm{dL}$. Antiglutamic acid decarboxylase antibody and anti-insulin auto-antibody tests were negative. Eruptive xanthoma or xanthelasma was not observed. There were no abnormalities, such as lipemia retinalis, found on an ophthalmologic examination. Abdominal computed tomography (CT) imaging revealed a diffuse edematous pancreas with adjacent fluid collection, which suggested AP of grade D (Fig. 1A). In addition, she had a fatty liver. No gallbladder involvement was seen.

Immediate management included intravenous rehydration therapy, continuous intravenous insulin infusion ( 6 units/hr), experimental antibiotics (piperacillin and tazobactam) for AP, and an analgesic ( $1 \mathrm{~g}$ of propacetamol) for pain control. On the second day of admission, her CRP level had increased to $33.47 \mathrm{mg} / \mathrm{dL}$ and the abdominal pain had persisted. She did not complain of steatorrhea. Follow-up CT imaging of the abdomen showed an increased volume of peripancreatic fluid collection relative to the previous examination suggesting AP of grade D (Fig. 1B). Her antibiotics were changed to broadspectrum antibiotics (meropenem and vancomycin). Four days after admission, her TG level had declined to $305 \mathrm{mg} / \mathrm{dL}$, and her CRP level was $16.41 \mathrm{mg} / \mathrm{dL}$. The abdominal pain had also resolved. Her blood culture was negative, and antibiotics were changed to piperacillin and tazobactam. She recovered from DKA, and her TG level decreased to $197 \mathrm{mg} / \mathrm{dL}$ without antilipid medication 7 days after admission. Because her abdominal pain had resolved and the serum amylase and lipase were nearly normal (27.2 and $68 \mathrm{U} / \mathrm{L}$, respectively), she commenced with a normal diet. Repeated ultrasonography showed resolution of pancreatic inflammation. She did not have complications of diabetes, including retinopathy, nephropathy, or neuropathy. Serial laboratory results related to HTG and AP during hospitalization are summarized in Table 1.

Given that her C-peptide level was not too low and considering her negative diabetes-related antibodies and high TG level, targeted gene panel sequencing was performed on the genes associated with diabetes and HTG. With informed consent from the patient, DNA was isolated from the peripheral blood leukocyte using the chemagic Magnetic Separation Module I

Table 1. Serial laboratory results

\begin{tabular}{lccccccc}
\hline Hospital day & $\mathrm{TG}(\mathrm{mg} / \mathrm{dL})$ & $\mathrm{TC}(\mathrm{mg} / \mathrm{dL})$ & $\mathrm{HDL}(\mathrm{mg} / \mathrm{dL})$ & $\mathrm{LDL}(\mathrm{mg} / \mathrm{dL})$ & Amylase $(\mathrm{U} / \mathrm{L})$ & $\mathrm{Lipase}(\mathrm{U} / \mathrm{L})$ & $\mathrm{CRP}(\mathrm{mg} / \mathrm{dL})$ \\
\hline At admission & 10,867 & 336 & 14 & 32 & 711.4 & $2,403.2$ & 1.61 \\
9 Hours after admission & 4,589 & 559 & 13 & 25 & 734.9 & $1,787.9$ & - \\
2 Days & 652 & - & - & - & 180.9 & 401.5 \\
4 Days & 305 & - & - & - & 59.4 & 107.2 & 16.41 \\
7 Days & 197 & - & - & - & 27.2 & 68 & 12.6 \\
10 Days & 227 & - & - & - & 40.4 & 114.2 \\
\hline
\end{tabular}

TG, triglyceride; TC, total cholesterol; HDL, high-density lipoprotein; LDL, low-density lipoprotein; CRP, C-reactive protein. 
method (PerkinElmer, Waltham, MA, USA). In total, 57,000 target exons of a total of 4,503 clinically relevant genes were captured by the xGen Inherited Disease Panel (Integrated DNA Technologies, Inc., Coralville, IA, USA) and sequenced with the NextSeq500 platform (Illumina, San Diego, CA, USA) for $2 \times 150$-bp paired-end reads, which were mapped to the hg19 utilizing the Burrow-Wheeler Aligner (ver. 0.7.12). For local realignment, recalibration, and variant-calling, the Genome Analysis Tool Kit (ver. 3.5) was used. We identified a heterozygous variant, c.4607C $>\mathrm{T}$ (p. Ala1537Val), in exon 38 of the ATP-binding cassette transporter subfamily $\mathrm{C}$ member 8 (ABCC8) for maturity-onset diabetes of the young (MODY) 12 , and no pathogenic variant was detected in other genes. The missense variant c.4607C $>\mathrm{T}$ (p.Ala1537Val) is considered a variant of unknown significance (VUS) based on the American College Medical Genetics and Genomics guidelines. This variant was found at a frequency of $0.0008 \%$ in a population database (gnomAD) and classified as being of "uncertain significance" in ClinVar. This variant was predicted to be "damaging" by algorithms developed to predict the effect of missense changes on protein structure (SIFT, Polyphen2, and MutationTaster). Generally, family segregation analysis can determine the pathogenicity of a VUS, but our patient's family members were not readily available for genetic testing and clinical information. After discharge, she was instructed to take basal insulin before breakfast and insulin aspart 3 times daily before meals.

\section{Discussion}

This case emphasizes the necessity for the recognition of AP associated with severe HTG in patients with DKA. DKA represents a state of severe insulin deficiency characterized by hyperglycemia and metabolic acidosis with ketone accumulations. ${ }^{5}$ The clinical presentations and complications of DKA result from hyperglycemia, dehydration, ketosis, and electrolyte imbalance. HTG in DKA is attributed to insulin deficiency, leading to increased lipolysis. Consequently, free fatty acid (FFA) is released, which activates the synthesis of veryLDL (VLDL). ${ }^{6,7)}$ Lipoprotein lipase (LPL) is responsible for the removal of VLDL and chylomicrons from the bloodstream. Insulin deficiency leads to decreased LPL activity, which causes HTG ${ }^{8)}$ Severe HTG is rare; however, it is an important risk factor for $\mathrm{AP}^{2)}$ Pancreatic lipase hydrolyzes TG, inducing the production of FFA, which activates trypsinogen and causes autodigestion of the pancreatic gland. ${ }^{9)}$ Major treatments of severe HTG include insulin, heparin, and plasmapheresis; however, few studies have enrolled children and adolescents to date. Maintaining a TG level of $<500 \mathrm{mg} / \mathrm{dL}$ is believed to result in symptom improvement. Insulin activates LPL, facilitating the degradation and clearing of TG. Heparin leads to the release of LPL from the endothelial cells, resulting in TG degradation. Oral antilipid medications are recommended when patients are capable of an oral diet, and fibrates can lower TG levels by $40 \%-60 \%{ }^{10)}$ In routine practice, the Friedewald equation is used to estimate LDL (total cholesterol [mg/dL] - HDL [mg/dL] $\mathrm{TG}[\mathrm{mg} / \mathrm{dL}] / 5)$. It has been believed that LDL is not exactly estimated when TG level exceeds $400 \mathrm{mg} / \mathrm{dL} .{ }^{11)}$ Generally, the Friedewald equation works properly in normolipidemia, not hyperlipidemia. Thus, there is a discrepancy between the LDL estimated by the equation and the actual measurements in our case. Published pediatric cases of AP associated with HTG in DKA are summarized in Table 2. All reports showed that severe HTG is an identifiable risk factor for AP. ${ }^{2)}$ TG levels gradually decreased to $<500 \mathrm{mg} / \mathrm{dL} 1-14$ days after management. In our patient, hydration and insulin infusion resulted in resolution of DKA and concurrent normalization of HTG. Initial lipid profiling is important in patients with DKA, because it can be a clue for timely abdominal imaging to ensure a diagnosis of AP. Moreover, abdominal pain is a common presentation of DKA as well as AP, with the symptom reported in nearly $50 \%$ of cases and related to variable gastrointestinal manifestations. ${ }^{12)}$

The $A B C C 8$ gene, encoding the sulfonylurea receptor 1

Table 2. Pediatric cases of acute pancreatitis associated with hypertriglyceridemia in diabetic ketoacidosis

\begin{tabular}{|c|c|c|c|c|c|c|}
\hline Study & Age (yr) & PeakTG (mg/dL) & Peak amylase (U/L) & Peak lipase (U/L) & $\begin{array}{l}\text { Management with } \\
\text { antilipid medication }\end{array}$ & $\begin{array}{l}\text { Time to normal TG } \\
\text { (day) } \\
\text { (TG level, mg/dL) }\end{array}$ \\
\hline Cywinski et al., 1965 ${ }^{18)}$ & 12 & $>1,000$ & 175 & NA & No & $7(232)$ \\
\hline Slyper et al., 1994 ${ }^{19)}$ & 14 & 3,119 & 627 & 3,680 & No & NA \\
\hline Hahn et al., 20109) & 20 & 15,000 & 443 & 615 & No & $3(506)$ \\
\hline Lutfi et al., 20125) & 10 & 16,334 & NA & 3,537 & $\begin{array}{c}\text { Fenofibrate, } \\
\text { plasmapheresis }\end{array}$ & $1.5(1,100)$ \\
\hline Aboulhosn and Arnason, $2013^{20)}$ & 18 & 1,724 & 319 & NA & No & NA \\
\hline Wolfgram and Macdonald, $2013^{3)}$ & 10 & 8,300 & NA & 2,950 & No & NA \\
\hline Singla et al., 2015 & 19 & 4,009 & 408 & 1,714 & Fenofibrate & $1(\mathrm{NA})$ \\
\hline Sharma et al., 2017) & 4 & 13,846 & 150 & 442 & No & $28(90)$ \\
\hline Zaher et al., 20197) & 14 & 6,400 & NA & 1,000 & $\begin{array}{c}\text { Fenofibrate, } \\
\text { unsaturated oils }\end{array}$ & $7(332)$ \\
\hline Yagnik et al., 2019 & 16 & 2,515 & 612 & 5,387 & Fenofibrate & $14(170)$ \\
\hline Our case & 14 & 10,867 & 711.4 & $2,403.2$ & No & $6(197)$ \\
\hline
\end{tabular}

TG, triglyceride; NA, not available. 
subunit of the ATP-sensitive potassium $\left(\mathrm{K}_{\text {ATP }}\right)$ channel, can regulate the secretion of insulin. ABCC 8 mutations have been shown to cause congenital hyperinsulinism (CHI), type $2 \mathrm{DM}$, gestational DM, neonatal diabetes, and MODY. ${ }^{13)}$ $\mathrm{CHI}$ is associated with the blindness of pancreatic $\beta$-cells responsible for insulin secretion, causing severe and persistent hypoglycemia. CHI is classified histologically into diffuse hyperinsulinism or focal islet-cell hyperplasia. Diffuse hyperinsulinism appears in an autosomal recessive manner, and entire $\beta$-cells in the pancreas are affected. On the other hand, its focal form is a heterozygous paternally inherited $K_{\text {ATP }}$ mutation of chromosome $11 \mathrm{p} 15$ region, which is confined to the islet cells of focal lesions. ${ }^{14)}$ It is challenging to differentiate MODY from other types of diabetes depending on clinical manifestations. In patients with MODY, $\beta$-cell function is generally conserved, and insulin is not required in the early stage of the disease. ${ }^{15}$ Kapoor et al. ${ }^{16)}$ reported a dominant $A B C C 8$ mutation, $\mathrm{A} 1537 \mathrm{~V}$, identified in our patient, which causes an asymptomatic carrier, hyperinsulinemic hypoglycemia, and gestational DM within 3 generations of a single family. Mutations in $A B C C 8$ can be associated with both hyperactivity and underactivity of the $\mathrm{K}_{\mathrm{ATP}}$ channel. Slow and progressive damage to $\beta$-cells owing to increased $\beta$-cell apoptosis can lead to both remission of hyperinsulinism and progression to diabetes. ${ }^{17)}$ Considering the clinical variability of $A B C C 8$ mutations, the mutation identified in our patient is suspected to be related to MODY 12 . As the result of target gene panel sequencing was classified as a VUS, family segregation analysis can enforce the pathogenicity of this variant. However, it was not possible to collect the detailed family history and conduct genetic testing of relatives in this case. This is the limitation of our case and thus, further validations in additional patients and functional studies are needed to prove the pathogenicity of this variant. Patients with MODY 12 respond to sulfonylurea therapy. ${ }^{13)}$ Thus, switching our patient from insulin to sulfonylurea is under consideration.

In this study, we presented a 14-year-old girl with AP related to severe HTG in DKA. To our knowledge, this is the first report of HTG-induced AP with DKA in a patient with MODY. In patients with DKA, timely awareness of severe HTG related to insulin deficiency is crucial for improving the consequence of AP. Based on our experience and the review of pertinent literature, we recommend considering AP in all DKA patients presenting with severe HTG to ensure early and proper management.

\section{Ethical statement}

Informed consent was obtained from the parents of the patient.

\section{Notes}

Conflicts of interest: No potential conflict of interest relevant to this article was reported.
Funding: This study was supported by a grant from Samsung Medical Center (\#GFO3200061).

Author contribution: Conceptualization: HP, SYC, EGY; Data curation: HP, EGY; Formal analysis: MSK, SML; Project administration: SYC, DKJ; Visualization: JK; Writing - original draft: HP; Writing - review \& editing: HP

ORCID

Hyojung Park: 0000-0002-1834-7446

Min-Sun Kim: 0000-0002-7799-3229

Jiyeon Kim: 0000-0002-6612-1732

Sae-Mi Lee: 0000-0002-0462-6700

Sung Yoon Cho: 0000-0003-2913-059X

Eun-Gyong Yoo: 0000-0002-6452-655X

Dong-Kyu Jin: 0000-0003-4162-2706

\section{References}

1. Edge JA, Hawkins MM, Winter DL, Dunger DB. The risk and outcome of cerebral oedema developing during diabetic ketoacidosis. Arch Dis Child 2001;85:16-22.

2. Rawla P, Sunkara T, Thandra KC, Gaduputi V. Hypertriglyceridemia-induced pancreatitis: updated review of current treatment and preventive strategies. Clin J Gastroenterol 2018;11:441-8.

3. Wolfgram PM, Macdonald MJ. Severe hypertriglyceridemia causing acute pancreatitis in a child with new onset type i diabetes mellitus presenting in ketoacidosis. J Pediatr Intensive Care 2013;2:77-80.

4. Wolfsdorf JI, Allgrove J, Craig ME, Edge J, Glaser N, Jain V, et al. ISPAD clinical practice consensus guidelines 2014 . Diabetic ketoacidosis and hyperglycemic hyperosmolar state. Pediatr Diabetes 2014;15 Suppl 20:154-79.

5. Lutfi R, Huang J, Wong HR. Plasmapheresis to treat hypertriglyceridemia in a child with diabetic ketoacidosis and pancreatitis. Pediatrics 2012;129:e195-8.

6. Sharma PK, Kumar M, Yadav DK. Severe hypertriglyceridemia causing pancreatitis in a child with new-onset type-i diabetes mellitus presenting with diabetic ketoacidosis. Indian J Crit Care Med 2017;21:176-8.

7. Zaher FZ, Boubagura I, Rafi S, Elmghari G, Elansari N. Diabetic ketoacidosis revealing a severe hypertriglyceridemia and acute pancreatitis in type 1 diabetes mellitus. Case Rep Endocrinol 2019;2019:8974619.

8. Singla AA, Ting F, Singla A. Acute pancreatitis secondary to diabetic ketoacidosis induced hypertriglyceridemia in a young adult with undiagnosed type 2 diabetes. JOP 2015;16:201-4.

9. Hahn SJ, Park JH, Lee JH, Lee JK, Kim KA. Severe hypertriglyceridemia in diabetic ketoacidosis accompanied by acute pancreatitis: case report. J Korean Med Sci 2010;25:1375-8.

10. Yagnik PJ, Desai PH, Modem VM. Hypertriglyceridemia with acute pancreatitis in pediatric diabetic ketoacidosis: a case report. Cureus 2019;11:e3844.

11. Martin SS, Blaha MJ, Elshazly MB, Brinton EA, Toth PP, 
McEvoy JW, et al. Friedewald-estimated versus directly measured low-density lipoprotein cholesterol and treatment implications. J Am Coll Cardiol 2013;62:732-9.

12. Umpierrez G, Freire AX. Abdominal pain in patients with hyperglycemic crises. J Crit Care 2002;17:63-7.

13. Lin L, Quan H, Chen K, Chen D, Lin D, Fang T. ABCC8related maturity-onset diabetes of the young (MODY12): a report of a chinese family. Front Endocrinol (Lausanne) 2020;11:645.

14. Hussain K, Flanagan SE, Smith VV, Ashworth M, Day $\mathrm{M}$, Pierro A, et al. An ABCC8 gene mutation and mosaic uniparental isodisomy resulting in atypical diffuse congenital hyperinsulinism. Diabetes 2008;57:259-63.

15. Hattersley AT, Greeley SAW, Polak M, Rubio-Cabezas O, Njølstad PR, Mlynarski W, et al. ISPAD clinical practice consensus guidelines 2018: the diagnosis and management of monogenic diabetes in children and adolescents. Pediatr Diabetes 2018;19 Suppl 27:47-63.
16. Kapoor RR, Flanagan SE, James CT, McKiernan J, Thomas AM, Harmer SC, et al. Hyperinsulinaemic hypoglycaemia and diabetes mellitus due to dominant $\mathrm{ABCC} 8 / \mathrm{KCNJ} 11$ mutations. Diabetologia 2011;54:2575-83.

17. Huopio H, Otonkoski T, Vauhkonen I, Reimann F, Ashcroft FM, Laakso M. A new subtype of autosomal dominant diabetes attributable to a mutation in the gene for sulfonylurea receptor 1. Lancet 2003;361:301-7.

18. Cywinski JS, Walker FA, White H, Traisman HS. Juvenile diabetes mellitus associated with acute pancreatitis. Acta Paediatr Scand 1965;54:597-602.

19. Slyper AH, Wyatt DT, Brown CW. Clinical and/or biochemical pancreatitis in diabetic ketoacidosis. J Pediatr Endocrinol 1994;7:261-4.

20. Aboulhosn K, Arnason T. Acute pancreatitis and severe hypertriglyceridaemia masking unsuspected underlying diabetic ketoacidosis. BMJ Case Rep 2013;2013:bcr2013200431. 